\title{
Long-Term Variability in the Winds of $\lambda$ Eridani and 66 Ophiuchi
}

\author{
Geraldine J. Peters \\ Space Sciences Center, University of Southern California, Los Angeles, \\ CA 90089-1341, USA
}

\begin{abstract}
.
Variations in the winds of the Be stars $\lambda$ Eri and 66 Oph during the lifetime of the IUE spacecraft and the response of the $\mathrm{H} \alpha$-emitting disk to these changes are briefly discussed. Common behavior is identified and evidence for cyclical variability on a time scale of $\sim 1 \mathrm{yr}$ is presented.
\end{abstract}

\section{Introduction}

Information on the nature of the long-term variability in Be star winds is important because it will aid us in determining the cause(s) for the mass loss in these objects. Any model or hypothesis proposed to explain the short-term (daily) activity in Be stars must also explain the long-term (month-year) wind behavior. Two B2e stars, $\lambda$ Eri and $66 \mathrm{Oph}$, were monitored with the IUE spacecraft throughout most of its the 17-year lifetime and provide us with a unique database on the long-term wind and photospheric variations in these typical Be stars. $\lambda$ Eri was observed from 1982-1995 (147 SWP HIRES images, 4 campaigns), while the wind behavior in 66 Oph was monitored from 1980-1995 (105 SWP HIRES images). The $\mathrm{H} \alpha$ emission in these stars was also contemporaneously monitored with the Coudé Feed and McMath telescopes at KPNO. Here we present some results on the behavior of the C IV wind line in both stars during the IUE era and discuss the response of the $\mathrm{H} \alpha$-emitting disk to the wind changes.

\section{2. $\lambda$ Eridani}

$\lambda$ Eridani is an active Be star that displays recurrent episodes of fairly weak $\mathrm{H} \alpha$ emission, various identifiable types of transient activity during periods of activity and quiescence (Smith 1989, and following series of papers), and a variable wind. The long-term IUE data have revealed that the wind indeed undergoes large variations. The equivalent width (EW) of the $\mathrm{C} I V$ wind line versus time is shown in Figure 1. The basal strength of the C IV wind line is $1.8 \AA$, but during an outburst it can reach $3.5 \AA$. When the wind is enhanced there is a prominent discrete absorption component (DAC) in C IV that displays an outflow velocity of $800-1000 \mathrm{~km} \mathrm{~s}^{-1}$ and a strength up to $0.30 \mathrm{EW}_{\text {tot }}$ (most of the gain in EW of C IV is in the DAC). Variations in the total wind absorption, absorption in the $\mathrm{DAC}$, and outflow velocity of the material in the region that produces the 


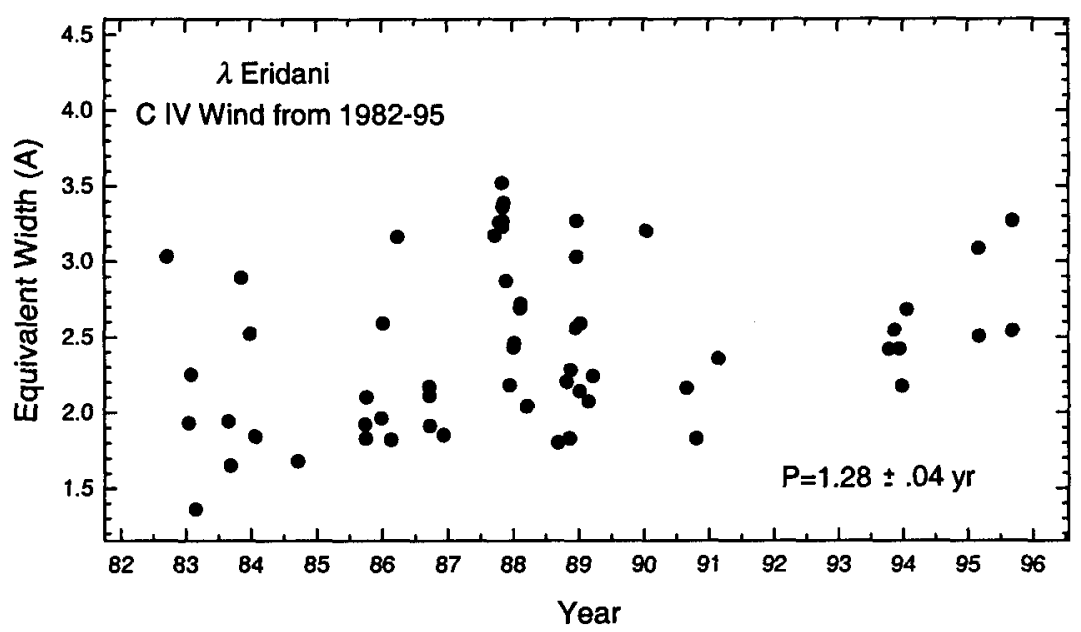

Figure 1. The EW of the C IV $\lambda 1550 \AA$ doublet in $\lambda$ Eri versus observation date. The data show seven outbursts that apparently recur every $\sim 1.3 \mathrm{yr}$.

DAC for three of the more well-covered activity cycles are shown in Peters (1998, Fig. 3). The DAC velocity tends to be more negative in the initial stages of an outburst $\left(<-1000 \mathrm{~km} \mathrm{~s}^{-1}\right)$.

The observations clearly show that epochs of enhanced wind are accompanied by episodes of $\mathrm{H} \alpha$ emission. Two outbursts with good IUE and $\mathrm{H} \alpha$ coverage (1987-88), shown in Figure 2, suggest that enhancement in the wind occurs before the growth of the Balmer emission.

Six discrete wind-H $\alpha$ outbursts have been identified in the time interval studied. Analysis of this data using a period search code (written by K. Bjorkman) based upon Scargle's (1982) algorithm suggests a timescale of $\sim 1.3 \mathrm{yr}$ for the activity episodes. The duration of an individual outburst varies somewhat from 0.1-0.4 yr and the major outburst of late 1987 appeared to be doublepeaked. The period found from the wind outbursts agrees with an optical photometric period $486^{\mathrm{d}}(1.33 \mathrm{yr})$ recently found by Mennickent et al. (1998).

\section{66 Ophiuchi}

The wind activity in 66 Oph is truly impressive (Grady et al. 1987, Peters 1988) as it spans the gamut from being strong (C IV EW 3.5 $\AA$ ) to essentially off (EW $<0.8 \AA$, mostly photospheric features)! The wind behavior from 1980-95 is shown in the upper panel of Figure 3. The corresponding activity in the $\mathrm{H} \alpha$ emission is displayed in the lower panel. Unlike $\lambda$ Eri, the wind in $66 \mathrm{Oph}$ usually displays multiple DACs. However, DAC velocities $<-1000 \mathrm{~km} \mathrm{~s}^{-1}$ seem to prevail, as in $\lambda$ Eri, in the early stages of an outburst (Peters 1988). Lowest DAC velocities are observed just before the wind fades. 


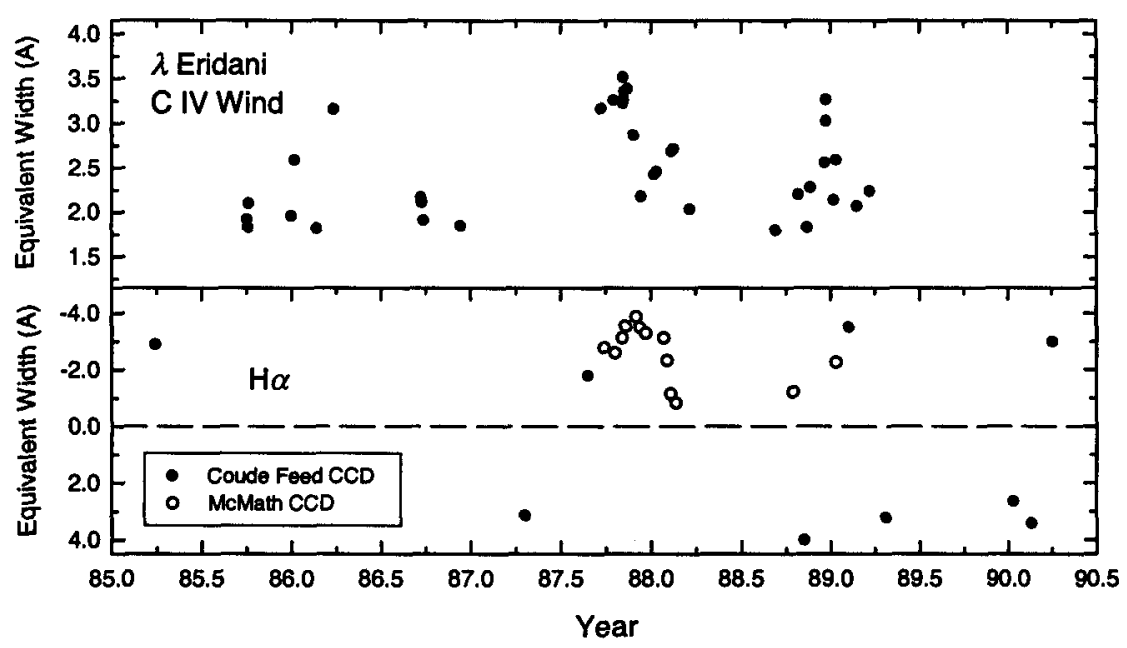

Figure 2. Contemporaneous IUE and ground-based CCD observations from Kitt Peak National Observatory show that an enhancement of the $\mathrm{H} \alpha$ emission accompanies but lags the strengthening of the wind. Some of the data from the KPNO McMath telescope are courtesy of M. Smith.

As in $\lambda$ Eri the intensity of the $\mathrm{H} \alpha$ emission appears to be correlated with the wind strength. Between 1988-90 there was a dramatic increase in the $\mathrm{H} \alpha$ emission that was accompanied by an increase in the strength of the wind, but the overall EW of the C IV $\lambda 1550$ was not as large as seen in the mid-1980s. There is evidence from a well-covered outburst in 1988 ( $\mathrm{H} \alpha$ data taken at the Ritter observatory by R. Dempsey) that the waxing/waning of the Balmeremitting disk lags the corresponding wind activity by $\sim 2$ months.

The time series analysis of the C IV data was inconclusive. Depending on the data that were included, the period search code suggested a period of about $1.0 \mathrm{yr}$ between $1980-87$ but $8-10 \mathrm{yr}$ overall. The wind appeared to be weaker in the 1990 s, but this could have been just a sampling problem.

\section{Common Behavior}

1. The IUE and ground-based observations demonstrate that the variability seen in the wind and Balmer lines results from a common physical mechanism that initiates a mass loss episode. The site of the mass loss on the photosphere feeds the disk. When the mass loss wanes, it takes about 1-2 months for substantial disk material to fall to the photosphere.

2. Most of the increase in wind absorption is in the form of DACs, and the largest outflow velocities $\left(\sim-1000 \mathrm{~km} \mathrm{~s}^{-1}\right)$ are seen prior to an outburst. Enhanced, high-velocity mass loss from the photosphere with subsequent 


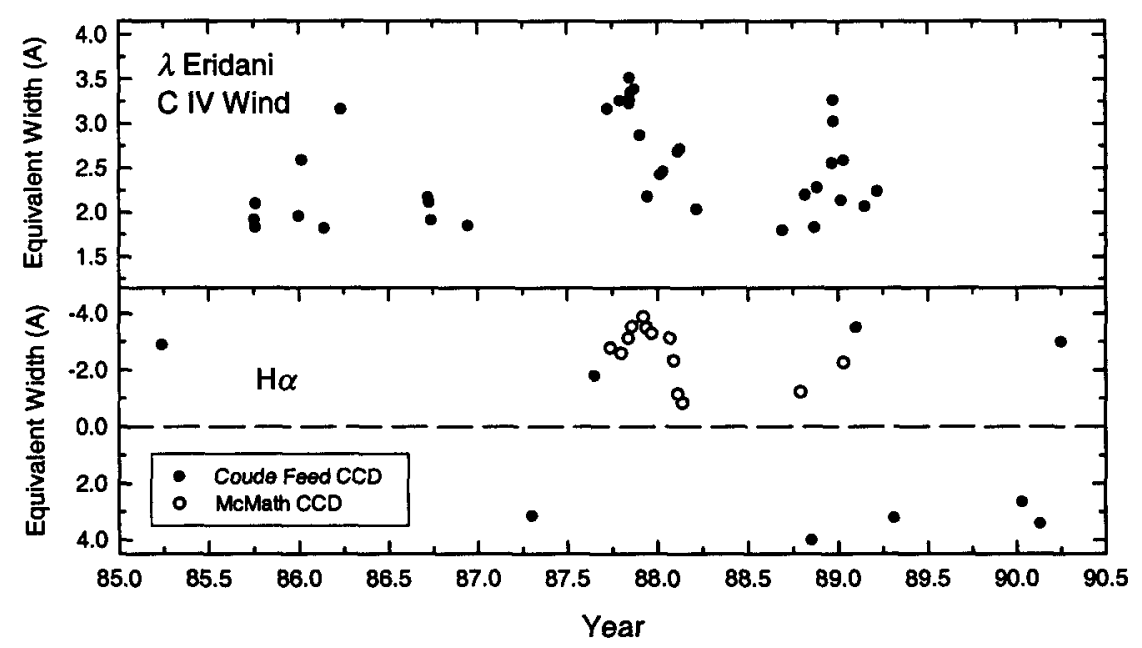

Figure 3. The EW of the C IV wind line and peak intensity of the $\mathrm{H} \alpha$ emission feature in $66 \mathrm{Oph}$ during the lifetime of the IUE satellite. Most of the CCD H $\alpha$ observations were obtained with the Coude Feed telescope at KPNO (resolution $\sim 0.2 \AA$ pixel $^{-1}$ ).

shocks in the wind characterize the outburst. There is need for detailed modeling of this behavior.

3. The outbursts are discrete and recur on a timescale of $\sim 1.3 \mathrm{yr}$ in $\lambda$ Eri. Some are short-lived (few weeks or less), while others last nearly a half year. In the 1980s, 66 Oph displayed recurrent discrete mass loss episodes every 1.0 year or so that seemed to be a precursor to its establishment of a massive Balmer-emitting disk in 1989-91. The IUE data cannot confirm that earlier wind behavior persisted into the 1990s.

I appreciate partial support from NASA grants NSG-5422 and NAG5-1296.

\section{References}

Grady, C.A., Sonneborn, G., Wu, C.-C., \& Henrichs, H. 1987, ApJS 65, 673

Mennickent, R.E., Sterken, C., \& Vogt, N. 1998, A\&A 330, 631

Peters, G.J. 1988, PASP 100, 207

Peters, G.J. 1998, in ESO Astrophys. Symp., Cyclical Variations in Stellar Winds, ed. L. Kaper \& A. Fullerton (Berlin: Springer), 127

Scargle, J.D. 1982, ApJ 263, 835

Smith, M.A. 1989, ApJS 71, 357 